\title{
Impaired neural processing of dynamic faces in left- onset Parkinson's disease
}

Citation for published version (APA):

Garrido-Vásquez, P., Pell, M. D., Paulmann, S., Sehm, B., \& Kotz, S. A. (2016). Impaired neural processing of dynamic faces in left-onset Parkinson's disease. Neuropsychologia, 82, 123-133. https://doi.org/10.1016/j.neuropsychologia.2016.01.017

Document status and date:

Published: 01/02/2016

DOI:

10.1016/j.neuropsychologia.2016.01.017

Document Version:

Publisher's PDF, also known as Version of record

Document license:

Taverne

Please check the document version of this publication:

- A submitted manuscript is the version of the article upon submission and before peer-review. There can be important differences between the submitted version and the official published version of record.

People interested in the research are advised to contact the author for the final version of the publication, or visit the DOI to the publisher's website.

- The final author version and the galley proof are versions of the publication after peer review.

- The final published version features the final layout of the paper including the volume, issue and page numbers.

Link to publication

\footnotetext{
General rights rights.

- You may freely distribute the URL identifying the publication in the public portal. please follow below link for the End User Agreement:

www.umlib.nl/taverne-license

Take down policy

If you believe that this document breaches copyright please contact us at:

repository@maastrichtuniversity.nl

providing details and we will investigate your claim.
}

Copyright and moral rights for the publications made accessible in the public portal are retained by the authors and/or other copyright owners and it is a condition of accessing publications that users recognise and abide by the legal requirements associated with these

- Users may download and print one copy of any publication from the public portal for the purpose of private study or research.

- You may not further distribute the material or use it for any profit-making activity or commercial gain

If the publication is distributed under the terms of Article $25 \mathrm{fa}$ of the Dutch Copyright Act, indicated by the "Taverne" license above, 


\title{
Impaired neural processing of dynamic faces in left-onset Parkinson's disease
}

\author{
Patricia Garrido-Vásquez ${ }^{\mathrm{a}, \mathrm{b}, *}$, Marc D. Pell $^{\mathrm{c}}$, Silke Paulmann ${ }^{\mathrm{d}}$, Bernhard Sehm ${ }^{\mathrm{a}}$, \\ Sonja A. Kotz ${ }^{\text {a,e }}$ \\ a Max Planck Institute for Human Cognitive and Brain Sciences, Leipzig, Germany \\ ${ }^{\mathrm{b}}$ Department of Experimental Psychology and Cognitive Science, Justus Liebig University Giessen, Giessen, Germany \\ ' School of Communication Sciences and Disorders, McGill University, Montreal, Canada \\ ${ }^{\mathrm{d}}$ Department of Psychology and Centre for Brain Science, University of Essex, Colchester, United Kingdom \\ e Department of Neuropsychology and Psychopharmacology, Maastricht University, Maastricht, The Netherlands
}

\section{A R T I C L E I N F O}

\section{Article history:}

Received 23 March 2015

Received in revised form 22 December 2015

Accepted 15 January 2016

Available online 15 January 2016

\section{Keywords:}

Parkinson's disease

Event-related potentials

Dynamic facial expressions

Emotion

Side of disease onset

\begin{abstract}
A B S T R A C T
Parkinson's disease (PD) affects patients beyond the motor domain. According to previous evidence, one mechanism that may be impaired in the disease is face processing. However, few studies have investigated this process at the neural level in PD. Moreover, research using dynamic facial displays rather than static pictures is scarce, but highly warranted due to the higher ecological validity of dynamic stimuli. In the present study we aimed to investigate how PD patients process emotional and nonemotional dynamic face stimuli at the neural level using event-related potentials. Since the literature has revealed a predominantly right-lateralized network for dynamic face processing, we divided the group into patients with left (LPD) and right (RPD) motor symptom onset (right versus left cerebral hemisphere predominantly affected, respectively). Participants watched short video clips of happy, angry, and neutral expressions and engaged in a shallow gender decision task in order to avoid confounds of task difficulty in the data. In line with our expectations, the LPD group showed significant face processing deficits compared to controls. While there were no group differences in early, sensory-driven processing (frontocentral N1 and posterior P1), the vertex positive potential, which is considered the fronto-central counterpart of the face-specific posterior N170 component, had a reduced amplitude and delayed latency in the LPD group. This may indicate disturbances of structural face processing in LPD. Furthermore, the effect was independent of the emotional content of the videos. In contrast, static facial identity recognition performance in LPD was not significantly different from controls, and comprehensive testing of cognitive functions did not reveal any deficits in this group. We therefore conclude that PD, and more specifically the predominant right-hemispheric affection in left-onset PD, is associated with impaired processing of dynamic facial expressions, which could be one of the mechanisms behind the often reported problems of PD patients in their social lives.
\end{abstract}

(c) 2016 Elsevier Ltd. All rights reserved.

\section{Introduction}

Parkinson's disease (PD) is more than a mere movement disorder. There is a plethora of work describing non-motor symptoms in $\mathrm{PD}$, for example, impaired executive functions or depression (for review see Kehagia et al. (2010)). It is also frequently reported that $\mathrm{PD}$ is associated with problems in the social domain, for example in face processing.

\footnotetext{
* Correspondence to: Justus Liebig University Giessen, Department of Experimental Psychology and Cognitive Science, Otto-Behaghel-Strasse 10F, 35394 Giessen, Germany.

E-mail address: patricia.garrido@psychol.uni-giessen.de (P. Garrido-Vásquez).
}

Previous studies have yielded evidence of impaired face processing in PD. Facial emotion recognition performance in PD patients is often lower than in matched healthy controls (e.g., Alonso-Recio et al., 2014; Ariatti et al., 2008; Clark et al., 2008, 2010; Sprengelmeyer et al., 2003; Suzuki et al., 2006; see also the meta-analysis by Gray and Tickle-Degnen (2010)). At the neural level, facial emotion discrimination may be altered in PD (Wieser et al., 2012). However, the evidence on facial emotion recognition in PD is still inconclusive, since several studies have failed to reveal deficits in PD (Pell and Leonard, 2005; Wieser et al., 2012; see also Péron et al. (2012), for a comprehensive review). Other lines of evidence report deficits in the recognition of facial identity in PD (Cousins et al., 2000; Dewick et al., 1991; Haeske-Dewick, 1996), in 
memory for faces (Kida et al., 2007), or in lip-reading from faces (Dewick et al., 1991; Haeske-Dewick, 1996).

So far, it is not clear which mechanisms may contribute to face processing deficits in PD. According to a study by Narme et al. (2011), PD patients are impaired at detecting changes of facial configurations (i.e., manipulations in the distance between facial features). Moreover, performance in this detection task significantly correlated with facial emotion recognition performance. Along similar lines, Marneweck and Hammond (2014) reported a significant correlation of visual form perception and emotional expression recognition in PD. Lotze et al. (2009) presented PD patients with video clips of neutral and emotional gestures, in which facial information was also visible. In fMRI, the authors observed diminished activations in several brain areas in PD, most notably in the visual motion area (V5), the fusiform gyrus, and the right superior temporal sulcus. Moreover, these reduced activations were also observed for neutral videos, suggesting that emotion-independent encoding of this kind of material might be impaired in PD. Thus, mounting evidence indicates that rather basic, emotion-independent processing of visual social information could be altered in PD, which could in turn also influence facial emotion processing (Beatty et al., 1989; Lotze et al., 2009; Marneweck and Hammond, 2014; Narme et al., 2011). Considering the neural bases of face processing may help elucidate how the disease could affect its underlying mechanisms.

One important characteristic of the human brain is hemispheric specialization, meaning that the right and left sides of the brain are, to a certain extent, functionally distinct. Studies on the neural underpinnings of face processing have revealed a wide-spread face processing network across the brain, in which the right hemisphere plays a relatively greater role than the left (Cattaneo et al., 2014; Kanwisher and Yovel, 2006). This has not only been shown for static, but also for dynamic facial displays (Thompson et al., 2007; Wheaton et al., 2004).

The functional significance of the right hemisphere in face processing also becomes evident when looking at studies with lesion patients. Patients with right-hemispheric lesions rated the emotion expressed in videos of fearful faces as less intense than a group of healthy participants, while the left-hemispheric lesion group did not differ from controls (Labudda et al., 2014). Righthemisphere lesions can also reduce performance in recognition tests for unfamiliar faces, recognition of familiar faces, or facial age attribution, while left-hemisphere lesions may lead to difficulty retrieving names of familiar faces (Carlesimo and Caltagirone, 1995). In PD, it is possible to distinguish between two subgroups with relatively greater neural degeneration in one hemisphere than the other. This is due to the unilateral onset of motor symptoms, and even though in the more advanced stages of the disease motor symptoms spread to the other side as well, the initially affected side remains the dominantly affected one in most cases. Conversely, neurodegeneration is stronger in the cerebral hemisphere contralateral to the more affected body side (Lee et al., 2015; Nahmias et al., 1985; Tatsch et al., 1997). Therefore, a distinction between PD patients with left-dominant motor symptoms (LPD) and those with right-dominant symptoms (RPD) could be useful to assess whether the relatively greater significance of the right hemisphere in face processing is reflected in impairments specifically in the LPD group.

While in most studies PD patients are not divided into subgroups according to motor symptom asymmetry, some previous work indicates that such division is indeed very useful, since LPD and RPD patients show distinct profiles of impairments. In visual global-local tasks LPD patients are more likely than RPD patients to show impairments at the global level rather than local, while in RPD it is vice versa (Schendan et al., 2009). This finding could also have implications for face processing, which is considered to rely on rather global, holistic processing (Kanwisher and Yovel, 2006). In line with the proposal to consider PD a disconnection syndrome, in which the less affected hemisphere gains dominance over the more degenerated one (Cronin-Golomb, 2010), the bigger picture in the literature suggests that visuospatial deficits are a prominent phenomenon in LPD (Amick et al., 2006; Karádi et al., 2015; Laudate et al., 2013, Lee et al., 2015; Tomer et al., 1993), while in RPD there is a tendency to be impaired in verbal tasks (Amick et al., 2006; Starkstein et al., 1987). Facial emotion recognition experiments have yielded equivocal results: LPD patients showed deficits in recognizing sad faces in one study (Ariatti et al., 2008), while anger recognition was compromised in two other studies (Clark et al., 2008, 2010). The same unclear pattern applies in RPD, with one study reporting impaired fear recognition (Ariatti et al., 2008) and others deficits in surprise (Clark et al., 2008, 2010). Three more studies do not report any asymmetry effects (Blonder et al., 1989; St. Clair et al., 1998; Ventura et al., 2012) and also failed to find facial emotion recognition impairments in PD. Thus, while the association between LPD and visuospatial impairments is relatively well established (Verreyt et al. (2011), for review), no clear picture has emerged to date with respect to emotional face processing and its relation to motor asymmetries in PD. It seems, however, plausible that the importance of the right hemisphere in face processing and the vulnerability of LPD patients to visuospatial deficits render this subgroup more likely than RPD to show face processing deficits, the origins of which could also lie in more generalized mechanisms of face processing rather than in emotion decoding per se.

Only few studies on emotional face processing have used dynamic facial expressions, whereas in daily life we normally encounter dynamic rather than static faces. Utilizing dynamic rather than static stimuli has been advocated particularly for patient studies, because these stimuli are more ecologically valid than photographs or drawings of faces (Garrido-Vásquez et al., 2011). Dynamic face stimuli lead to more wide-spread neural activation patterns, most notably in temporal regions linked to processing socially relevant stimuli (Sato et al., 2004; Trautmann et al., 2009). They also go along with enhanced behavioral emotion recognition rates (Ambadar et al., 2005; Bould and Morris, 2008). Importantly, this pattern may apply to PD patients as well (Kan et al., 2002, but see Paulmann and Pell (2010)), and therefore more investigation using dynamic face stimuli in PD is warranted. The previously mentioned PD study by Lotze et al. (2009) used videos of emotional gestures, and thus dynamic visual stimuli. Even though the focus of that study was on emotional and non-emotional gestures, facial expressions were simultaneously visible in the videos. Interestingly, all but one PD patient in the study had lower dopamine transporter availability in the right rather than the left basal ganglia (and the right-left difference was statistically significant), which would correspond to LPD. Therefore, the study indirectly relates LPD to impaired processing of dynamic, socially relevant visual material, which also includes emotionally neutral expressions. Thus, more research into the mechanisms of processing socially relevant, dynamic visual stimuli in PD is needed, and a division of the patient sample into LPD and RPD seems useful based on previous evidence.

Regarding the neural encoding of faces measured with ERPs, two characteristic components can be observed during the first $200 \mathrm{~ms}$ of face processing. These have been described as dipolar complexes due to opposing polarities at fronto-central compared to posterior electrode sites (Luo et al., 2010; Rossion et al. 1999): the first component peaking approximately $100 \mathrm{~ms}$ after stimulus onset is of positive polarity at posterior electrodes and negative at fronto-central sites (termed P1 and N1, respectively). It is assumed that coarse analysis of low-level stimulus features takes place at this stage (Luo et al., 2010). This dipolar N1/P1 complex is 
followed by another dipolar complex characteristic to face processing: the posterior N170 and the simultaneous vertex positive potential (VPP) at fronto-central electrodes, with peak latencies between 140 and $180 \mathrm{~ms}$ after stimulus onset (Joyce and Rossion, 2005). During this processing step, structural processing of a face takes place, and the brain distinguishes between faces and other visual objects (Bentin et al., 1996). VPP and N170 very likely reflect the same brain process, and their amplitudes vary depending on which reference is applied to the EEG data (Joyce and Rossion, 2005; Rellecke et al., 2013). Even though these components have been associated with structural face processing, their amplitudes may be modulated by facial expression as well (Hinojosa et al., 2015).

To the best of our knowledge, only two studies so far have reported ERP data on emotional faces in PD. One study focused on neural generators of ERPs and reported diminished amygdala responses underlying the N1 to fearful faces in PD (Yoshimura et al., 2005). Another study, which focused on posterior electrodes, did not reveal any P1 or N170 alterations in PD, but emotion discrimination at later stages was impaired (Wieser et al., 2012). However, none of these studies used dynamic facial expressions and none differentiated between LPD and RPD. A recent EEG study reported reduced interhemispheric coherence during dynamic audio-visual emotion processing in PD (Yuvaraj et al., 2014), which is in line with the description of PD as a disconnection syndrome (Cronin-Golomb, 2010).

In most facial emotion recognition tasks, a verbal label has to be assigned to a facial expression. This is also referred to as explicit emotion processing (Paulmann et al., 2008) or high-level appraisal processing (Bach et al., 2008) in the literature. On the other hand, there is implicit or low-level appraisal processing, which can be induced with more shallow tasks such as gender decisions. There are some differences between the two task types: firstly, it has been argued that implicit tasks are closer to natural processing environments than explicit ones (Paulmann et al., 2008), since we normally analyze emotional signals from our conversation partners in an implicit way. Secondly, evidence suggests that especially explicit tasks may involve the basal ganglia (Paulmann et al., 2008), which would also render PD patients to be more susceptible to show impairments in these tasks. Thirdly, the relation between explicit tasks and cognitive impairments must be considered. Not only are cognitive impairments a common symptom in PD (Kehagia et al., 2010), but also some direct relations between cognitive status and performance in explicit emotion tasks have been shown in PD (Pell and Leonard, 2003). Therefore, we decided to employ an implicit, shallow task in order to avoid these potential confounds in the ERP data. Moreover, the face-sensitive N170 component is more pronounced in implicit rather than explicit tasks, according to metaanalytic evidence (Hinojosa et al., 2015).

To sum up, the present study aimed to investigate the time course of dynamic emotional face processing in LPD and RPD compared to a group of matched healthy controls. Basic underlying questions to this study were whether LPD or RPD patients, or both, show alterations during the first $200 \mathrm{~ms}$ of face processing, during which the two previously described dipolar complexes are observed in the ERP (i.e., fronto-central N1/posterior $\mathrm{P} 1$ and fronto-central VPP/posterior N170), and if yes, whether these alterations are modulated by emotion or not. To shed light on these issues we conducted an ERP study, in which we presented participants with emotional (happy and angry) and neutral dynamic facial expressions. To avoid contamination of the ERPs by explicit emotion recognition tasks, which may be cognitively demanding for PD patients and additionally tap executive functions (Pell and Leonard, 2003), we decided to use a shallow and very easy gender decision task, only to ensure that participants were attending to the stimuli. In light of previous evidence we hypothesized that the LPD group would be more likely than the RPD group to show alterations in face processing. Based on previous reports of early neural emotion discrimination deficits in LPD in the auditory domain (Garrido-Vásquez et al., 2013) and possible impairments of basic face processing mechanisms in that group, we further predicted that emotion-related deficits would also be more likely in LPD than RPD.

\section{Methods}

\subsection{Participants}

Twenty-four individuals diagnosed with idiopathic PD and 12 age- and education-matched healthy controls participated in the present study. Twelve patients had a left-sided disease onset and also reported motor symptoms predominantly on the left side of the body at the time of testing (named LPD throughout); the other half displayed a right onset and a current rightward asymmetry of motor symptoms (named RPD throughout). Two RPD patients were excluded from the sample. In one case, this was due to an unstable motor symptom lateralization pattern, with medical records showing right-sided disease onset, but stronger left-sided motor symptoms at the time of testing. The other right-onset patient was withdrawn from the sample because of an exceptionally low performance (13 out of 27 points, close to the chance level of 11.5 points) in the Benton Facial Recognition Test (Benton et al., 1983). Latter test was used to screen for basic facial recognition abilities, with all remaining participants scoring 16 points and higher. (We are aware that the score of 16 points, which was achieved by one RPD patient, would be classified as an impaired score. However, withdrawing this specific patient from the RPD group did not significantly alter the data and was therefore not done due to power considerations.)

Informed consent was obtained from all participants prior to testing, and the study was approved by the Ethics Committee at the University of Leipzig. All study-related procedures were in accordance with the Declaration of Helsinki. Participants were paid for their participation.

All study participants had normal or corrected-to-normal visual acuity according to self-report and all were right-handed as assessed by the Edinburgh Handedness Inventory (Oldfield, 1971). None of the participants reported any history of psychiatric or neurological disorders (except PD in patients), and none were taking any psychotropic medication. All participants scored in the non-clinical range of the Beck Depression Inventory (BDI; Beck et al., 1961), with a maximum score of 18 points. The Mini-Mental Status Examination (MMSE; Folstein et al., 1975) was used as a screening tool for dementia, with all participants scoring in the normal range of 26 points or higher out of a maximum of 30 . Since PD may sometimes lead to mild hemispatial neglect (Lee et al., 2001), two screening procedures targeting neglect symptoms were also applied. These were Albert's Line Cancellation Test (Albert, 1973) and a clock-drawing test. None of the participants experienced any problems in these two tasks.

All PD patients were evaluated with the Unified Parkinson's Disease Rating Scale (UPDRS; Fahn and Elton, 1987) by an experienced neurologist, who also determined disease severity according to Hoehn and Yahr stages (Hoehn and Yahr, 1967). All were on anti-Parkinsonian medication during the measurements. Seven LPD patients and five RPD patients were taking levodopa, while the remaining ten patients were medicated with dopamine agonists and MAO B inhibitors only. The daily levodopa-equivalent dose was calculated according to the formula previously used by MacDonald et al. (2011). The upper part of Table 1 provides 
Table 1

Comparison of demographic and disease-specific variables and test scores between the three groups.

\begin{tabular}{|c|c|c|c|c|}
\hline Variable & Controls & LPD & RPD & $\begin{array}{l}\text { Group } \\
\text { effect }^{\mathrm{a}}\end{array}$ \\
\hline \multicolumn{5}{|l|}{ Demographic variables } \\
\hline Age in years & $66.17(8.79)$ & $65.50(7.81)$ & $\begin{array}{l}66.30 \\
(6.40)\end{array}$ & $p>.91$ \\
\hline Range & $49-75$ & $53-74$ & $56-75$ & \\
\hline $\begin{array}{l}\text { Years of formal } \\
\text { education }\end{array}$ & $14.23(1.71)$ & $14.17(2.68)$ & $\begin{array}{l}14.40 \\
(4.01)\end{array}$ & $p>.90$ \\
\hline Male/female & $7 / 5$ & $6 / 6$ & $7 / 3$ & $p>.63^{\mathrm{b}}$ \\
\hline \multicolumn{5}{|l|}{ Disease-specific variables } \\
\hline Duration in years & - & $5.83(4.13)$ & $4.10(1.60)$ & $p>.27$ \\
\hline Range & & $2-17^{c}$ & $1-6$ & \\
\hline Hoehn \& Yahr stage ${ }^{d}$ & - & 2 (range: $1-3$ ) & $\begin{array}{l}2 \text { (all in } \\
\text { stage } 2 \text { ) }\end{array}$ & $p>.13^{b}$ \\
\hline $\begin{array}{l}\text { Daily levodopa-equiva- } \\
\text { lent dose in } \mathrm{mg}\end{array}$ & - & $430(332)$ & $371(263)$ & $p>.75$ \\
\hline Total UPDRS motor score & - & $22.33(9.19)$ & $\begin{array}{l}19.00 \\
(8.11)\end{array}$ & $p>.17$ \\
\hline Left motor score & - & $9.33(3.50)$ & $3.80(2.53)$ & $\boldsymbol{p}<.001$ \\
\hline Right motor score & - & $4.92(3.40)$ & $8.40(3.66)$ & $p=.018$ \\
\hline \multicolumn{5}{|l|}{ Test scores } \\
\hline MMSE & $29.33(1.07)$ & $28.75(1.14)$ & $\begin{array}{l}29.10 \\
(0.74)\end{array}$ & $p>.29$ \\
\hline BDI & $5.58(3.68)$ & $9.25(5.48)$ & $7.80(5.77)$ & $p>.32$ \\
\hline Benton facial recognition & $22.33(1.72)$ & $21.33(2.42)$ & $21.5(2.72)$ & $p>.49$ \\
\hline Digit Span forward & $10.08(1.78)$ & $9.83(2.08)$ & $9.90(1.79)$ & $p>.90$ \\
\hline Digit Span backward & $6.67(1.92)$ & $6.50(1.38)$ & $6.20(1.14)$ & $p>.98$ \\
\hline Count letters & $9.67(0.49)$ & $9.50(0.67)$ & $9.80(0.42)$ & $p>.52$ \\
\hline Rearranging task & $7.33(0.89)$ & $7.17(0.72)$ & $6.70(1.42)$ & $p>.33$ \\
\hline Listening span & $4.13(0.68)$ & $4.25(0.97)$ & $3.30(0.75)$ & $p=.028$ \\
\hline \multicolumn{5}{|l|}{$\begin{array}{l}\text { Trail-Making Test com- } \\
\text { pletion time in } \\
\text { seconds }\end{array}$} \\
\hline Part A & 33.67 (7.39) & 36.00 (11.09) & $\begin{array}{l}42.90 \\
(9.07)\end{array}$ & $p=.041$ \\
\hline Part B & $71.67(11.70)$ & $82.25(25.41)$ & $\begin{array}{l}90.90 \\
(45.52)\end{array}$ & $p>.61$ \\
\hline \multicolumn{5}{|l|}{ Word fluency } \\
\hline Test 1 & $13.42(4.78)$ & $13.50(4.70)$ & $\begin{array}{l}11.80 \\
(4.13)\end{array}$ & $p>.52$ \\
\hline Test 2 & $16.67(3.34)$ & $14.75(3.57)$ & $\begin{array}{l}14.40 \\
(5.34)\end{array}$ & $p>.23$ \\
\hline Test 3 & $16.67(5.09)$ & $18.33(4.60)$ & $\begin{array}{l}18.30 \\
(5.81)\end{array}$ & $p>.84$ \\
\hline
\end{tabular}

The table shows means (standard deviations in brackets) for demographic and disease-specific variables and test scores in the three groups. The rightmost column shows whether there was a significant main effect of group for each of the variables.

${ }^{a}$ Nonparametric Kruskal-Wallis test (or Mann-Whitney test when comparing two groups) unless otherwise stated.

b Chi-square test.

${ }^{c}$ One patient in the LPD group had a disease duration of 17 years, while among the other LPD patients maximum disease duration was 8 years. See also Section 2.1 for further information.

${ }^{\mathrm{d}}$ Median value.

information on demographic and disease-specific variables in the three groups. Please note that the wide range of disease duration in the LPD group (2-17 years) is due to one single patient with very long disease duration. Without this patient, maximum disease duration in the LPD group is 8 years. Excluding this specific patient from the LPD group did not alter the pattern of ERP results and was therefore not considered necessary.

\subsection{Neuropsychological tests}

Before the actual experiment, all participants were invited to a neuropsychological testing session. In addition to the tests mentioned in the participants section, we assessed working memory and executive functions. Working memory was tested using the forward and backward digit span (Wechsler, 1997), a letter counting task (a mixed sequence of numbers and letters is presented and the number of letters in each sequence has to be reported), and a rearranging task (a mixed sequence of numbers and letters is presented and their order has to be changed). The letter counting and rearranging tasks are described in more detail elsewhere (Pagonabarraga et al., 2008). Furthermore, an in-house listening span task was used (auditory version of the reading span by Daneman and Carpenter (1980), translated into German). To get an estimate of executive functions, we applied parts $A$ and $B$ of the Trail-Making Test (Reitan, 1992) and three word fluency measures, each assessed during one minute (1: phonemic fluency-generate as many words as possible starting with the letter "T"; 2 : alternating phonemic-semantic fluency: alternate between words starting with the letter " $\mathrm{S}$ " and names of countries starting with any letter; 3: action verb fluency: generate as many action verbs as possible). 


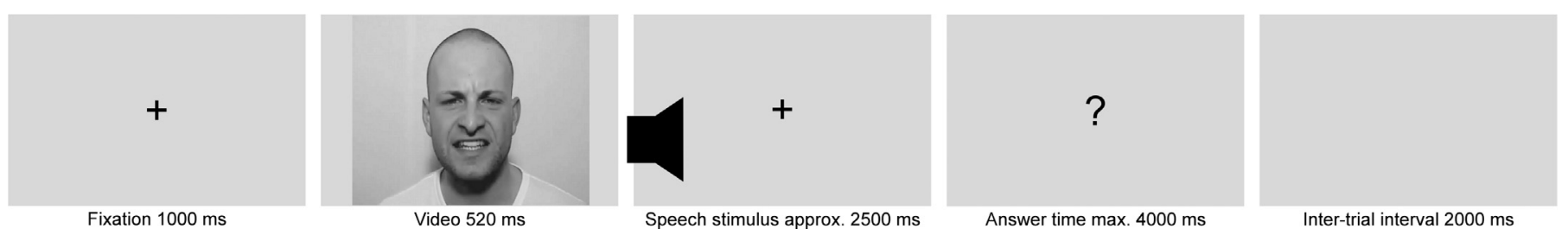

Fig. 1. Scheme of one exemplary trial in the experiment. See the also Section 2.3 for a detailed description.

\subsection{Stimuli and procedures}

Black-and-white video stimuli of facial expressions were used. The videos were recorded from four semi-professional actors (two females), who produced emotional sentences in a happy, an angry, and a neutral tone of voice while showing the corresponding facial expressions. The audio track was removed from the videos and fragments of $520 \mathrm{~ms}$ duration were cut out from the original videos. To avoid different emotion recognition points in the video fragments, these were cut out from the middle of the original videos, in which the full-blown facial expressions were visible. Additionally, the first author checked manually that in each video fragment the intended emotion could already be recognized in the first video frame. The actor's gaze was directed toward the observer in all videos, and mouth movements were visible. Videos were cropped and/or centered when necessary to ensure that the faces appeared in the middle of the screen and were of comparable size across all videos. This was adjusted and measured using a still picture of each video's first frame. As the actors did not perform major head movements during the recordings, head position remained approximately constant during the course of a video. Image resolution was $720 \times 576$ pixels. The MPEG- 4 codec was used to allow for optimized video timing. Twenty-five frames per second were presented. We used Presentation software (Neurobehavioral Systems, San Francisco, USA) for experimental control.

Video stimuli were first rated by a sample of 28 healthy participants, who did not participate in the present study. Three of them had to be excluded due to low performance (more than two standard deviations below the group mean for at least one emotional category). The remaining 25 participants ( 12 females) had a mean age of 25.48 years $(S D=2.63$ years) and reported normal or corrected-to-normal visual acuity. For each of the four actors, 120 trials (40 per emotional category) were presented, adding up to 480 trials. Each video fragment had to be categorized as happy, angry, neutral, or unclear/other within four seconds after its offset. The percent-correct rates from this study were used to create rank lists for each speaker and emotion category. The 12 most easily recognized stimuli for each of the four actors and three emotion categories, respectively, were then selected for the present study (144 stimuli in total). Mean recognition rates for the videos used in the present study were: anger $99 \%$, happiness $97 \%$, and neutral $82 \%$.

The EEG experiment was conducted in an electrically shielded, sound-attenuated and dimly lit room with participants sitting at a viewing distance of about $100 \mathrm{~cm}$ from the computer screen. The videos were presented centrally, with faces subtending a visual angle of approximately three degrees to each side. Participants were instructed to indicate the actor's gender by means of a button press. Half of the participants pressed the left button for "female" and the right button for "male", while the other half proceeded vice versa.

The course of one experimental trial is depicted in Fig. 1. Trials started with a black fixation cross on a gray background matched to the mean luminance of the video stimuli. Fixation lasted for one second and was followed by the video presentation for $520 \mathrm{~ms}$.
Immediately after the end of the video, the fixation cross was presented again and an auditory pseudo-speech stimulus in happy or angry intonation, produced by the same actor previously depicted in the video, was transmitted via loudspeakers. These utterances had a mean length of $2.5 \mathrm{~s}$ and were presented to investigate priming effects of emotionally congruent or incongruent facial expressions on the processing of auditory speech intonation (data not reported here). Then, the fixation cross changed into a question mark prompting a participant's gender decision response. Response time was limited to a maximum of four seconds. After the button press, or after four seconds had timed out in the event of no response being registered, a blank screen was presented for two seconds before the next trial started. The experiment consisted of a total of 144 trials, which were presented in a pseudorandomized order that differed for each participant. A break was included after every 48 trials.

\subsection{EEG recording and data analysis}

The EEG was recorded from $27 \mathrm{Ag} / \mathrm{AgCl}$ scalp electrodes mounted in an elastic cap and included the following locations according to the extended 10-20 system: FP1, FP2, F7, F3, FZ, F4, F8, FT7, FC3, FC4, FT8, T7, C3, CZ, C4, T8, CP5, CP6, P7, PO7, P3, PZ, $\mathrm{P} 4, \mathrm{P} 8, \mathrm{PO} 8, \mathrm{O} 1$, and $\mathrm{O} 2$. Acquisition was carried out with a bandpass between DC and $250 \mathrm{~Hz}$ at a sampling rate of $500 \mathrm{~Hz}$. The ground electrode was placed on the sternum. Four additional electrodes were placed on bilateral outer canthi and above and below the right eye to record eye movements. Electrode resistance was kept below five $\mathrm{k} \Omega$. An average reference was used during the measurement, and electrodes were re-referenced to the average of left and right mastoids offline. A bandpass filter was applied to the data offline $(0.2-30 \mathrm{~Hz}, 4363$ points, Hamming window). We used an ocular correction procedure (Pfeifer et al., 1995) to eliminate eye movement artifacts and thus increase the number of trials eligible for statistical analysis. All EEG data processing procedures were accomplished with the EEP software package (Max Planck Institute for Human Cognitive and Brain Sciences, Leipzig, Germany).

ERPs were time-locked to the video onset, with a $100 \mathrm{~ms}$ prestimulus baseline, and averaged across the whole video duration of $520 \mathrm{~ms}$. As ERPs at fronto-central and posterior electrodes are of opposite polarity in early visual processing, separate ANOVAs were conducted for fronto-central and posterior electrode sites. Frontocentral electrodes were grouped into the following three regions: left anterior (F3, FC3), right anterior (F4, FC4), and midline (FZ, CZ). Two posterior regions were formed as follows: left posterior (PO7, $\mathrm{O} 1)$ and right posterior (PO8, O2). We defined two time windows of interest based on visual inspection of the ERP data. To analyze $\mathrm{N} 1$ at fronto-central and P1 at posterior electrodes, the data were averaged over a time window from 70 to $110 \mathrm{~ms}$ post stimulus onset. The second time window extended from 130 to $190 \mathrm{~ms}$, corresponding to the VPP at fronto-central sites and the N170 posteriorly. The ANOVA followed a 3 (emotion) $\times 3$ (region) $\times 3$ (group) design at fronto-central electrode sites, while in the case of posterior sites the region factor was twofold ( $3 \times 2 \times 3$ design).

To check for more generalized group differences beyond face 


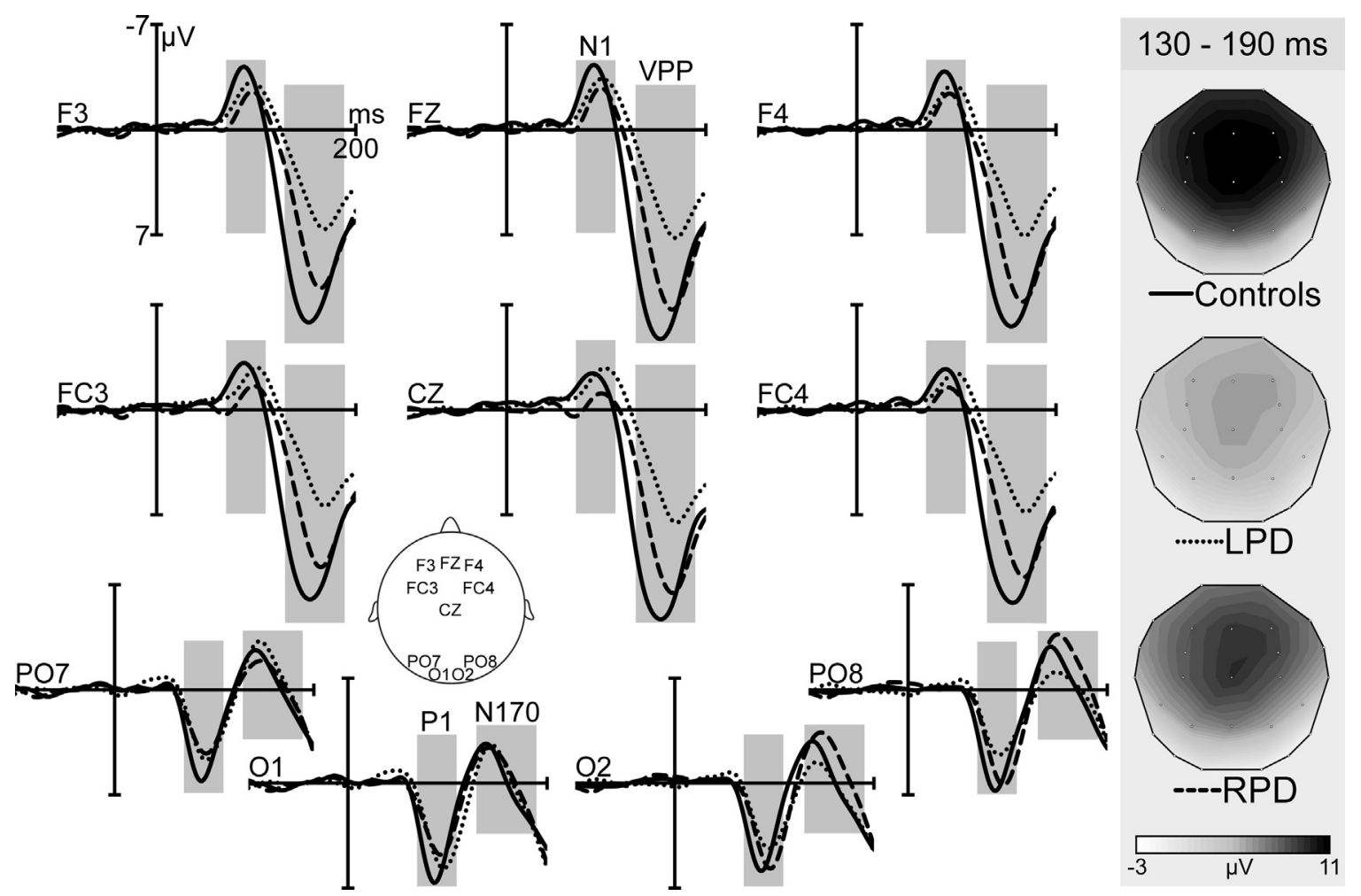

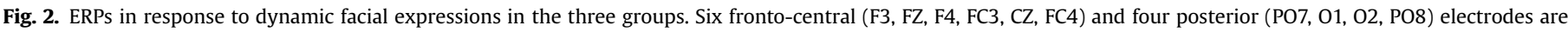

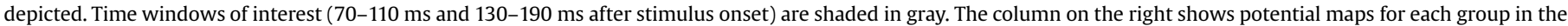
VPP/N170 time window.

processing, we also analyzed auditory N100 and P200 for the happy and angry pseudo-speech sentences presented after each face stimulus. Time windows were defined from 90 to 150 (N100) and 180 to 280 (P200) ms after stimulus onset by means of visual data inspection. EEG data processing was identical to the procedure described above, and the first $100 \mathrm{~ms}$ of the auditory stimulus served as in-stimulus baseline. A 2 (emotion) $\times 5$ (region) $\times$ 3 (group) ANOVA was calculated on auditory ERPs.

Only significant results $(p<.05)$ are reported in the results unless otherwise stated. Greenhouse-Geisser corrected $p$-Values were used where necessary. Only correctly responded to and artifact-free trials were used for analysis (24\% of the trials were excluded on average). An emotion $\times$ group ANOVA with the number of trials as a dependent variable indicated no significant group differences as to how many trials were included into the ERP analysis ( $p s>.58$ ). As expected, behavioral performance was at ceiling in all groups ( $>98 \%$ correct), confirming that the task was in fact very easy. Moreover, responses were given at a fixed point in time. Therefore, behavioral data were not further analyzed. Statistical calculations were carried out with SAS software (SAS Institute, Cary, USA).

\section{Results}

\subsection{Demographic variables, disease-specific variables, and test} scores

Table 1 shows a comparative overview of demographic and disease-specific variables as well as test scores in the three groups. As can be seen, the groups did not significantly differ with respect to demographic variables, and the two patient groups were not significantly different regarding the disease-specific measures of disease duration, the total UPDRS motor score, the daily levodopa- equivalent dose, or the Hoehn and Yahr stage. An expected significant group difference emerged for both left and right motor scores.

Importantly, the three groups did not significantly differ in their recognition of static faces, measured with the Benton Facial Recognition Test, $H(2)=1.43, p>.4$. With respect to other test scores, the listening span test $[H(2)=6.80, p=.028]$ as well as part A of the Trail-Making Test $[H(2)=6.29, p=.041]$ yielded a significant main effect of group. Follow-up tests of these significant results (non-parametric Mann-Whitney tests) revealed that RPD patients performed significantly worse than controls in both listening span, $U(1)=80.5, Z=2.29, p=.009$, and the Trail-Making Test part $A, U(1)=151.0, Z=2.34, p=.008$. Furthermore, LPD patients outperformed the RPD group in both tests [listening span: $U$ $(1)=82.0, Z=2.17, p=.011$; Trail-Making Test part A: $U(1)=144.5$, $Z=1.92, p=.028$ ], but were not significantly different from controls. All the other tests did not reveal any significant group differences (see Table 1).

\subsection{Event-related potentials in response to dynamic facial expressions}

Event-related potentials at the six fronto-central and the four posterior electrodes used for data analysis are displayed graphically in Fig. 2.

\subsubsection{Fronto-central $N 1$ and posterior P1}

Analysis of the $\mathrm{N} 1$ component at fronto-central electrodes and of the P1 component at posterior sites yielded only one significant effect, the region $\times$ group interaction in the $\mathrm{P} 1, F(2,31)=3.37$, $p=.047, \eta_{\mathrm{p}}{ }^{2}=.179$. Further analysis of this finding revealed a significant main effect of region in the RPD group, $F(1,9)=10.57$, $p=.01, \eta_{\mathrm{p}}{ }^{2}=.540$, manifested in a larger P1 amplitude at right posterior compared to left posterior electrodes. In the LPD and HC 
groups no significant main effect of region emerged ( $p s>.1$ ).

\subsubsection{Fronto-central VPP and posterior N170}

In the VPP, there was a significant main effect of group, $F$ $(2,31)=7.89, p=.002, \eta_{\mathrm{p}}{ }^{2}=.337$. According to the post-hoc Tukey test, the LPD group's VPP amplitude $(M=4.92 \mu \mathrm{V}, S D=2.94)$ was significantly smaller than that of controls $(M=11.08 \mu \mathrm{V}, S D=4.86)$ at an alpha level of $p=.002$. In the RPD group, VPP amplitude $(M=8.69 \mu \mathrm{V}, S D=3.62)$ was also lower than in controls and higher than in LPD. Regarding the statistical difference between RPD and LPD, there was a trend towards significance $(p=.071)$, while controls and RPD did not differ $(p>.3)$. There was a group-independent main effect of region, $F(2,62)=27.12, \quad p<.0001$, $\eta_{\mathrm{p}}{ }^{2}=.467$. VPP amplitudes were higher at the right than at the left fronto-central region, $F(1,31)=9.77, p=.004, \eta_{\mathrm{p}}{ }^{2}=.228$. VPP amplitude at midline electrodes was also higher than on the left, $F$ $(1,31)=48.58, p<.0001, \eta_{\mathrm{p}}{ }^{2}=.595$.

In addition to amplitude differences, visual inspection of the VPP indicated latency variation between the three groups. Therefore, VPP peak latency was calculated for all participants. It was quantified as the highest positive peak in a time window from 120 to $240 \mathrm{~ms}$ after stimulus onset, and it was extracted separately from each of the the six fronto-central electrodes for each participant and condition. The ANOVA on VPP peak latency revealed a significant main effect of group, $F(2,31)=5.15, p=.012, \eta_{\mathrm{p}}{ }^{2}=.250$. Mean VPP latency was $152 \mathrm{~ms}(S D=11)$ in controls, $163 \mathrm{~ms}$ $(S D=12)$ in RPD patients, and $172 \mathrm{~ms}(S D=21)$ in LPD patients. According to the post-hoc Tukey test comparing the three groups, the difference between controls and LPD patients was significant at an alpha level of $p<.009$, while the LPD and RPD groups $(p>.2)$, as well as RPDs and controls $(p>.3)$ did not differ.

Concerning the N170, the counterpart of the VPP at posterior electrodes, no significant main effects of group or interactions with this factor were observed $(p s>1)$. There was a significant emotion $\times$ region interaction $F(2,62)=4.13, p=.021$. However, follow-up analyzes of this effect did not yield any significant results ( $p s>.3$ ). Due to the group differences in VPP latency, the N170 component was also analyzed for latency differences between the groups. Mean peak latency of the $\mathrm{N} 170$ was $141 \mathrm{~ms}(S D=17)$ in controls, $145 \mathrm{~ms}(S D=15)$ in the LPD group, and $148 \mathrm{~ms}(S D=14)$ in the RPD group. The ANOVA on N170 latencies did not yield any significant results involving the group factor $(p s>.1)$.

To sum up, significant group differences were found in the VPP component. The LPD group exhibited both lower VPP amplitudes and delayed VPP latencies compared to controls, and they showed

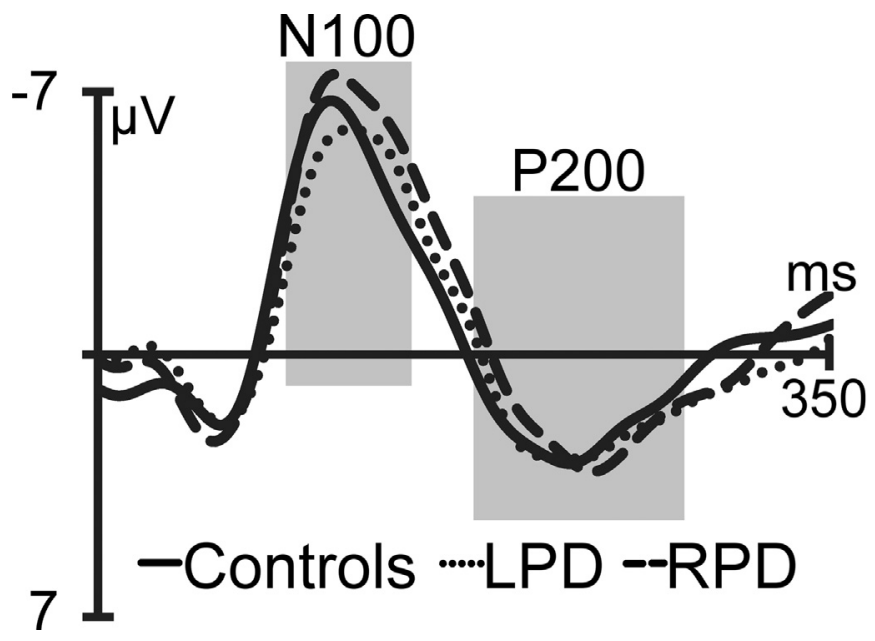

Fig. 3. ERPs in response to auditory pseudo-speech stimuli at one central electrode (CZ). The time windows in which N100 and P200 were analyzed are shaded in gray. a trend towards lower VPP amplitudes than the RDP group. Since there were no significant interactions with the factor emotion, the alterations in the LPD group seem to be emotion-independent. The RPD group did not significantly differ from controls in the VPP. However, this group showed lower P1 amplitudes at left compared to right electrode sites, in line with the relatively greater lefthemispheric neural degeneration in this group.

\subsection{Relation of VPP results to disease-specific variables and test scores}

Spearman correlations were computed on the collapsed sample of patients to test for possible relations between VPP amplitude or latency and the disease-specific variables of disease duration, total motor score, asymmetry index (calculated as [left motor scoreright motor score]/[left motor score + right motor score]), and daily levodopa-equivalent dose. The correlation between asymmetry index and VPP amplitude was significant and persisted at trend level upon Bonferroni correction, $r=-0.50, p=.072$, indicating that the stronger the asymmetry of motor symptoms toward the left side of the body, the smaller the amplitude of the VPP.

We created composite scores for working memory (backward digit span, rearranging task, listening span) and executive functions (Trail-Making Test A and B, word fluency tests) to test for relationships between VPP and cognitive variables. These composite scores reduced the number of possible correlations. For an exact description of the procedure see Garrido-Vásquez et al. (2013). According to the Kruskal-Wallis test, the three groups did not significantly differ on the composite scores ( $p s>.09$ ), even though numerically the lowest performance was observed in RPD for both the working memory and the executive functions score.

Correlations were computed on the whole sample $(N=34)$ to test for possible relations between VPP latency or amplitude and BDI score, Benton Facial Recognition Test score, and the two composite scores. Bonferroni-corrected p-values revealed that the executive functions composite score was negatively correlated with VPP latency, $r=-.45, p=.028$.

\subsection{Event-related potentials in response to emotionally intoned pseudo-speech}

To ensure that the group differences we observed in dynamic face processing were not due to more generalized alterations of neural functioning or processing difficulties for dynamic social stimuli, we analyzed the ERPs elicited by the vocal emotional stimuli, which followed face presentation. No significant main effects of group or interactions with this factor were found in the auditory N100 or P200 ( $p s>.13$ ). For a graphical display of these data, please refer to Fig. 3.

\section{Discussion}

In the present study we aimed to investigate the time course of dynamic emotional face processing in PD at the neural level, using the high temporal resolution of ERPs. Furthermore, we took into account the heterogeneity among patients in terms of motor symptom asymmetry. We observed that LPD patients, whose right hemisphere is predominantly affected by neural degeneration, exhibited impairments during the first $200 \mathrm{~ms}$ of face processing. This was reflected in a temporally delayed VPP and a diminished VPP amplitude, while alterations at an earlier processing stage (N1/P1) were not observed in LPD. VPP alterations turned out to be independent of emotion and thus seem to represent a generalized face encoding problem in LPD patients, irrespective of the emotion expressed. Moreover, a dissociation between ERPs and behavioral 
measures was revealed, because LPD patients were not impaired in the Benton Facial Recognition Test. Furthermore, the reported alterations are unlikely to be explained by cognitive deficits in LPD, because this group was not significantly different from controls in any of the acquired cognitive measures. There were also no significant group effects in the auditory ERPs, which discourages alternative explanations such as general cognitive slowing or more unspecific problems in processing dynamic social information in LPD. Furthermore, due to the very easy and shallow task we used, confounds in the ERP data due to task difficulty are very unlikely. Dynamic face processing deficits in LPD would be of high relevance, considering the impact these may have in everyday life.

The finding of impaired face processing in the LPD group is in line with the literature linking face processing predominantly to the right hemisphere (Cattaneo et al., 2014; Kanwisher and Yovel, 2006), which also holds true for dynamic facial expressions (Thompson et al., 2007; Wheaton et al., 2004), and with the localization of the neural generators underlying the VPP or N/M170 components (Luo et al., 2010; Rossion et al., 2003; Gao et al., 2013). Moreover, lesion studies have shown that right- but not lefthemispheric lesions go along with impaired face processing (Carlesimo and Caltagirone, 1995; Labudda et al., 2014). Thus, the present findings in a patient group with right-hemispheric degeneration (LPD) add up to this evidence and outline once more the important role of the right hemisphere already at the initial stages of face processing. Furthermore, the correlation between motor symptom asymmetry and VPP amplitude, even though only at trend level after Bonferroni correction, strengthens the link between lateralization of motor symptoms to the left side of the body and face processing impairments.

The role of the right superior temporal sulcus (STS) may be important to consider in the present study, because it is thought of as a key region for coding biological motion (Grossman and Blake, 2002), and tends to be more activated by dynamic facial expressions than by dynamic yet non-social stimuli (Wheaton et al., 2004). Interestingly, Lotze et al. (2009) reported diminished right STS activation in PD to dynamic gesture stimuli, which were presented together with the corresponding dynamic facial expressions. Since all but one patient in Lotze et al.'s sample exhibited lower dopamine transporter availability in the right basal ganglia, the study provides indirect evidence of a link between LPD and diminished right STS activation in the processing of socially relevant, dynamic visual stimuli. Moreover, EEG activity in the VPP/ N170 time window significantly correlates with activation in the right STS for upright faces, with higher ERP amplitudes related to stronger activation in this area (Nguyen and Cunnington, 2014; see also Sadeh et al. (2010), for a similar result), while correlations of N170 amplitude with activity in other classical face processing regions (fusiform face area, occipital face area, and medial frontal gyrus) were not significant (Nguyen and Cunnington, 2014). Therefore, diminished right STS activity could also provide a plausible explanation for our LPD data.

Our finding of a generalized face processing impairment in PD, potentially of structural nature, is also in line with previous studies on the link between basic visual processes and emotional face processing in PD. Narme et al. (2011) showed that PD patients are behaviorally impaired at detecting changes of face configurations, and detection performance was significantly correlated with emotion recognition. Conversely, Marneweck and Hammond (2014) showed a significant correlation between visual form perception and facial emotion recognition in PD. While the methodology used in these studies was not suitable to reveal the exact time course of face processing impairments, the present data indicate that an early stage, at which basic features such as facial structure are encoded, is affected. Our results moreover show that it makes sense to divide the patient group according to the asymmetry of motor symptoms, since the subgroups may show distinct profiles of PD-related impairments. Accordingly, in other PD work measuring the ERP response to static faces, VPP latency was delayed for about $13 \mathrm{~ms}$ on average in PD, but this difference was not significant (Kida et al., 2007). We argue that this may be due to heterogeneity among the PD sample (which is apparent in the standard deviations in Kida et al.'s study), because we could report significant VPP alterations only for the LPD group, but not for the RPD group. A few previous studies had already indicated that LPD patients may be more affected by visuospatial deficits than RPD patients, which presumably has to do with the role of the right hemisphere in visuospatial functions (Cronin-Golomb, 2010). Particularly, mild hemispatial neglect in terms of a rightward visual bias has been repeatedly associated with LPD (Laudate et al., 2013; Lee et al., 2001). In the present study, we used two test procedures to screen for hemispatial neglect in the participants, who all performed normally. Therefore, we would not consider hemispatial neglect a prime interpretation of our results, but since these symptoms tend to be very mild and hard to detect, we cannot fully discard this possibility either. In general, it is still unclear whether and how potential visuospatial impairments in LPD would relate to processing impairments for dynamic visual stimuli of social relevance. However, despite the differences between LPD and RPD patients, which were also confirmed in the present study, the RPD group's VPP amplitude was descriptively also smaller and peaked later than in healthy controls. Therefore, one may speculate that with further disease progression, which also goes along with increasing neurodegeneration in the nondominantly affected right hemisphere, deficits in dynamic face processing could become evident in RPD.

Our result of impaired early face processing in LPD is unlikely explained by cognitive deficits in patients. Firstly, the LPD groupin contrast to the RPD group-was not significantly different from healthy controls in any of the cognitive measures (see Table 1 ) or in the composite scores. Secondly, the only significant correlation was found between the executive functions composite score and VPP latency, but there were no significant correlations with VPP amplitude. Thus, the data fail to show a consistent association between cognitive status on the one hand, and the processing of dynamic facial expressions on the other. However, this pattern also indicates that even in the absence of cognitive decline in PD, deficits in the social domain may be present and make everyday interactions more difficult.

Our results may seem at odds with those of Wieser et al. (2012) on emotional face processing in PD, since these authors did not find any group differences during the first $200 \mathrm{~ms}$ of face processing. However, one major difference between their study and ours is the distinction between LPD and RPD. As argued before, using a unitary PD patient sample, group differences may be missed if they are only present in a subgroup. Moreover, Wieser et al. (2012) employed static photographs; hence the comparability between the two studies may be limited. We suggest that the dynamics inherent in our stimuli may be key to the face encoding deficits in LPD observed in the present study, based on the apparent dysfunction of the right STS and visual motion area (V5) in LPD (Lotze et al., 2009). According to a set of studies in which PD patients were significantly impaired at recognizing vocal emotion while facial emotion recognition from static pictures was intact (Pell and Leonard, 2003, 2005), the authors suggested that functional breakdowns in processing communicative stimuli could become evident especially in the case of dynamic information, due to the involvement of the basal ganglia in timing processes and their 
importance for decoding information that extends over temporal domains. Conversely, LPD patients in our study performed well on the Benton Facial Recognition Test, thus-at least with respect to this specific test-static face processing was intact, contrary to other findings in PD (Cousins et al., 2000; Dewick et al., 1991; Haeske-Dewick, 1996). Moreover, there was no significant correlation between performance in this test and VPP amplitude or latency. Therefore, it is not clear whether this test is a good indicator for the processing of dynamic facial expressions. However, even though we consider the dynamic nature of our stimuli to be a key factor in the results we report here, there is some debate in the literature whether face-specific ERP components in the time range of VPP/N170 are affected by the dynamic nature of stimuli or not (for a positive result, see Puce et al. (2000); for a negative result, see Recio et al. (2011)). Future PD studies should directly compare ERPs in response to static versus dynamic emotional and non-emotional expressions. In general, it would be important to apply more dynamic facial expressions in research, due to their higher ecological validity and their frequent occurrence in real life.

From a methodological point of view it may seem interesting that the significant effects in our study were observed in the VPP, but not in the N170. As outlined in the introduction, these two face-sensitive components occur simultaneously in the ERP, and they are supposed to reflect the same underlying neural mechanisms (Joyce and Rossion, 2005). However, their amplitudes vary with reference placement, and the mastoids reference used in the present study favors the VPP (Joyce and Rossion, 2005), while N170 is maximal if an average reference is used (Joyce and Rossion, 2005; Rellecke et al., 2013). Since average reference requires at least 32 scalp electrodes to be measured (Pivik et al., 1993), we did not compute it in the present study. Thus, it is very likely that we observed significant effects in the VPP rather than in the N170 because of the mastoids reference we used.

It is also important to note that in the auditory ERPs no significant group differences emerged. These results are in line with data from a previous study (Garrido-Vásquez et al., 2013), which showed that with the exception of disgust, vocal emotion processing is not impaired in LPD if pseudo-speech stimuli are presented instead of natural speech. The absence of differences between the LPD group and healthy controls both in the auditory task and during an initial processing stage (N1/P1) of dynamic facial expressions in the present study strengthens the interpretation of an impairment of face-specific processing in LPD.

Even though on the basis of our data we argue that LPD is associated with an emotion-independent deficit for basic mechanisms of face processing, probably of structural nature, we cannot discard the possibility that emotion-specific effects could be observed in the N1/P1 or VPP/N170 components if more emotional categories were included. In fact, the ERP literature reports early emotion effects in face perception at a very early point in time, namely in the time window of N1/P1 (e.g., Jetha et al., 2012; Luo et al., 2010). Such early emotion effects may occur when stimuli are highly salient, as in the case of fear or anger expressions (Adolphs, 2002). Fear was not included in the present study, and the anger stimuli we used were probably not salient enough to trigger such early neural modulations, considering that they were continuous and dynamic, while most ERP studies use static pictures of facial expressions. Normally static picture stimuli represent peak emotion and may therefore be perceived as more intense and salient compared to their dynamic counterparts. Regarding the VPP/N170 time window, a recent meta-analysis also reported evidence for a modulation of ERP amplitude by emotion (Hinojosa et al., 2015). Future studies should extend the present findings by including more emotional categories, and by comparing the processing of static and dynamic displays of emotion in PD directly.

\section{Conclusion}

The present ERP study on dynamic emotional face processing in PD revealed that LPD patients show emotion-independent impairments during the first $200 \mathrm{~ms}$ of processing dynamic faces, reflected in a temporally delayed and attenuated VPP component. These results are in line with the predominant role assigned to the right hemisphere in (dynamic) face processing and in the generation of the VPP/N170 ERP components. Furthermore, our study once more shows that a division of PD patients into subgroups is very useful to better describe heterogeneous disease profiles. Lastly, we would like to outline that deficits in the social domain should be taken more into account in PD and can be present despite intact cognitive functioning.

\section{Acknowledgments}

The authors are grateful to the participants of this study for their contributions. We would also like to thank Cornelia Schmidt for recruiting the healthy controls and for assistance in data acquisition. Furthermore, we thank Anne-Kathrin Franz for administrative help during the course of this study and Elizabeth Kelly for proofreading. Financial support for this study was provided by the Canadian Institutes of Health Research [Grant number CIHR\#MOP62867 to M.D.P. and S.A.K.] and the Parkinson Society Canada [Grant number MOP 62868 to M.D.P. and S.A.K.]. The funding sources had no role in study design, data collection, data analysis, data interpretation, writing this article, or in the decision to publish this work. We also gratefully acknowledge the many helpful comments by three anonymous reviewers on an earlier version of this manuscript.

\section{References}

Adolphs, R., 2002. Recognizing emotion from facial expressions: psychological and neurological mechanisms. Behav. Cogn. Neurosci. Rev. 1, 21-62.

Albert, M.L., 1973. A simple test of visual neglect. Neurology 23, 658-664.

Alonso-Recio, L., Martín, P., Rubio, S., Serrano, J.M., 2014. Discrimination and categorization of emotional facial expressions and faces in Parkinson's disease. J. Neuropsychol. 8, 269-288.

Ambadar, Z., Schooler, J.W., Cohn, J.F., 2005. Deciphering the enigmatic face: the importance of facial dynamics in interpreting subtle facial expressions. Psychol. Sci. 16, 403-410.

Amick, M.M., Grace, J., Chou, K.L., 2006. Body side of motor symptom onset in Parkinson's disease is associated with memory performance. J. Int. Neuropsychol. Soc. $12,736-740$.

Ariatti, A., Benuzzi, F., Nichelli, P., 2008. Recognition of emotions from visual and prosodic cues in Parkinson's disease. Neurol. Sci. 29, 219-227.

Bach, D.R., Grandjean, D., Sander, D., Herdener, M., Strik, W.K., Seifritz, E., 2008. The effect of appraisal level on processing of emotional prosody in meaningless speech. Neuroimage 42, 919-927.

Beatty, W.W., Goodkin, D.E., Weir, W.S., Staton, R.D., Monson, N., Beatty, P.A., 1989. Affective judgments by patients with Parkinson's disease or chronic progressive multiple sclerosis. Bull. Psychon. Soc. 27, 361-364.

Beck, A.T., Ward, C.H., Mendelson, M., Mock, J., Erbaugh, J., 1961. An inventory for measuring depression. Arch. Gen. Psychiatry 4, 561-571.

Bentin, S., Allison, T., Puce, A., Perez, E., McCarthy, G., 1996. Electrophysiological studies of face perception in humans. J. Cogn. Neurosci. 8, 551-565.

Benton, A.L., Sivan, A.B., Hamsher, K.S., Varney, N.R., Spreen, O., 1983. Benton Facial Recognition Test. Oxford University Press, New York.

Blonder, L.X., Gur, R.E., Gur, R.C., 1989. The effects of right and left hemiparkinsonism on prosody. Brain Lang. 36, 193-207.

Bould, E., Morris, N., 2008. Role of motion signals in recognizing subtle facial expressions of emotion. Br. J. Psychol. 99, 167-189. 
Carlesimo, G.A., Caltagirone, C., 1995. Components in the visual processing of known and unknown faces. J. Clin. Exp. Neuropsychol. 17, 691-705.

Cattaneo, Z., Renzi, C., Bona, S., Merabet, L.B., Carbon, C.C., Vecchi, T., 2014. Hemispheric asymmetry in discriminating faces differing for featural or configural (second-order relations) aspects. Psychon. Bull. Rev. 21, 363-369.

Clark, U.S., Neargarder, S., Cronin-Golomb, A., 2008. Specific impairments in the recognition of emotional facial expressions in Parkinson's disease. Neuropsychologia 46, 2300-2309.

Clark, U.S., Neargarder, S., Cronin-Golomb, A., 2010. Visual exploration of emotional facial expressions in Parkinson's disease. Neuropsychologia 48, 1901-1913.

Cousins, R., Hanley, J.R., Davies, A.D., Turnbull, C.J., Playfer, J.R., 2000. Understanding memory for faces in Parkinson's disease: the role of configural processing Neuropsychologia 38, 837-847.

Cronin-Golomb, A., 2010. Parkinson's disease as a disconnection syndrome. Neuropsychol. Rev. 20, 191-208.

Daneman, M., Carpenter, P., 1980. Individual differences in working memory and reading. J. Verbal Learn. Verbal Behav. 19, 450-466.

Dewick, H.C., Hanley, J.R., Davies, A.D., Playfer, J., Turnbull, C., 1991. Perception and memory for faces in Parkinson's disease. Neuropsychologia 29, 785-802.

Fahn, S., Elton, R.L., 1987. Members of the UPDRS development committee. Unified Parkinson's disease rating scale. In: Fahn, S., Marsden, C.D., Calne, D.B., Goldstein, M. (Eds.), Recent Developments in Parkinson's Disease. Macmillan Health Care Information, Florham Park, NJ, pp. 153-164.

Folstein, M.F., Folstein, S.E., McHugh, P.R., 1975. Mini-mental state: a practical method for grading the cognitive state of patients for the clinician. J. Psychiatr. Res. 12, 189-198.

Gao, Z., Goldstein, A., Harpaz, Y., Hansel, M., Zion-Golumbic, E., Bentin, S., 2013. A magnetoencephalographic study of face processing: M170, gamma-band oscillations and source localization. Hum. Brain Mapp. 34, 1783-1795.

Garrido-Vásquez, P., Jessen, S., Kotz, S.A., 2011. Perception of emotion in psychiatric disorders: on the possible role of task, dynamics, and multimodality. Soc. Neurosci. 6, 515-536.

Garrido-Vásquez, P., Pell, M.D., Paulmann, S., Strecker, K., Schwarz, J., Kotz, S.A., An, E.R.P., 2013. Study of vocal emotion processing in asymmetric Parkinson's disease. Soc. Cogn. Affect. Neurosci. 8, 918-927.

Gray, H.M., Tickle-Degnen, L., 2010. A meta-analysis of performance on emotion recognition tasks in Parkinson's disease. Neuropsychology 24, 176-191.

Grossman, E.D., Blake, R., 2002. Brain areas active during visual perception of biological motion. Neuron 35, 1167-1175.

Haeske-Dewick, H.C., 1996. Are perception and memory for faces influenced by a specific age at onset factor in Parkinson's disease? Neuropsychologia 34, 315-320.

Hinojosa, J.A., Mercado, F., Carretié, L., 2015. N170 sensitivity to facial expression: a meta-analysis. Neurosci. Biobehav. Rev. 55, 498-509.

Hoehn, M.M., Yahr, M.D., 1967. Parkinsonism: onset, progression and mortality. Neurology 17, 427-442.

Jetha, M.K., Zheng, X., Schmidt, L.A., Segalowitz, S.J., 2012. Shyness and the first $100 \mathrm{~ms}$ of emotional face processing. Soc. Neurosci. 7, 74-89.

Joyce, C., Rossion, B., 2005. The face-sensitive N170 and VPP components manifest the same brain processes: the effect of reference electrode site. Clin. Neurophysiol. 116, 2613-2631.

Kan, Y., Kawamura, M., Hasegawa, Y., Mochizuki, S., Nakamura, K., 2002. Recognition of emotion from facial, prosodic and written verbal stimuli in Parkinson's disease. Cortex 38, 623-630.

Kanwisher, N., Yovel, G., 2006. The fusiform face area: a cortical region specialized for the perception of faces. Philos. Trans. R. Soc. B: Biol. Sci. 361, 2109-2128.

Karádi, K., Lucza, T., Aschermann, Z., Komoly, S., Deli, G., Bosnyák, E., Kovács, 2015. Visuospatial impairment in Parkinson's disease: the role of laterality. Laterality 20, 112-127.

Kehagia, A.A., Barker, R.A., Robbins, T.W., 2010. Neuropsychological and clinical heterogeneity of cognitive impairment and dementia in patients with Parkinson's disease. Lancet Neurol. 9, 1200-1213.

Kida, Y., Tachibana, H., Takeda, M., Yoshikawa, H., Okita, T., 2007. Recognition memory for unfamiliar faces in Parkinson's disease: behavioral and electrophysiologic measures. Park. Relat. Disord. 13, 157-164.

Labudda, K., Mertens, M., Steinkroeger, C., Bien, C.G., Woermann, F.G., 2014. Lesion side matters-an fMRI study on the association between neural correlates of watching dynamic fearful faces and their evaluation in patients with temporal lobe epilepsy. Epilepsy Behav. 31, 321-328.

Laudate, T.M., Neargarder, S., Cronin-Golomb, A., 2013. Line bisection in Parkinson's disease: investigation of contributions of visual field, retinal vision, and scanning patterns to visuospatial function. Behav. Neurosci. 127, 151-163.

Lee, A.C., Harris, J.P., Atkinson, E.A., Fowler, M.S., 2001. Disruption of estimation of body-scaled aperture width in Hemiparkinson's disease. Neuropsychologia 39, 1097-1104.

Lee, E.Y., Sen, S., Eslinger, P.J., Wagner, D., Kong, L., Lewis, M.M., Huang, X., 2015. Side of motor onset is associated with hemisphere-specific memory decline and lateralized gray matter loss in Parkinson's disease. Park. Relat. Disord. 21, 465-470.

Lotze, M., Reimold, M., Heymans, U., Laihinen, A., Patt, M., Halsband, U., 2009. Reduced ventrolateral fMRI response during observation of emotional gestures related to the degree of dopaminergic impairment in Parkinson disease. J. Cogn. Neurosci. 21, 1321-1331.
Luo, W., Feng, W., He, W., Wang, N.Y., Luo, Y.J., 2010. Three stages of facial expression processing: eRP study with rapid serial visual presentation. Neuroimage 49, 1857-1867.

MacDonald, P.A., MacDonald, A.A., Seergobin, K.N., Tamjeedi, R., Ganjavi, H., Provost J.S., Monchi, O., 2011. The effect of dopamine therapy on ventral and dorsal striatum-mediated cognition in Parkinson's disease: support from functional MRI. Brain 134, 1447-1463.

Marneweck, M., Hammond, G., 2014. Discriminating facial expressions of emotion and its link with perceiving visual form in Parkinson's disease. J. Neurol. Sci. 346, 149-155.

Nahmias, C., Garnett, E.S., Firnau, G., Lang, A., 1985. Striatal dopamine distribution in Parkinsonian patients during life. J. Neurol. Sci. 69, 223-230.

Narme, P., Bonnet, A.M., Dubois, B., Chaby, L., 2011. Understanding facial emotion perception in Parkinson's disease: the role of configural processing. Neuropsychologia 49, 3295-3302.

Nguyen, V.T., Cunnington, R., 2014. The superior temporal sulcus and the N170 during face processing: single trial analysis of concurrent EEG-fMRI. Neuroimage 86, 492-502.

Oldfield, R.C., 1971. The assessment and analysis of handedness: the Edinburgh inventory. Neuropsychologia 9, 97-113.

Pagonabarraga, J., Kulisevsky, J., Llebaria, G., García-Sánchez, C., Pascual-Sedano, B. Gironell, A., 2008. Parkinson's disease-cognitive rating scale: a new cognitive scale specific for Parkinson's disease. Mov. Disord. 23, 998-1005.

Paulmann, S., Pell, M.D., 2010. Dynamic emotion processing in Parkinson's disease as a function of channel availability. J. Clin. Exp. Neuropsychol. 32, 822-835.

Paulmann, S., Pell, M.D., Kotz, S.A., 2008. Functional contributions of the basal ganglia to emotional prosody: evidence from ERPs. Brain Res. 1217, 171-178.

Pell, M.D., Leonard, C.L., 2003. Processing emotional tone from speech in Parkinson's disease: a role for the basal ganglia. Cogn. Affect. Behav. Neurosci. 3, 275-288.

Pell, M.D., Leonard, C.L., 2005. Facial expression decoding in early Parkinson's disease. Cogn. Brain Res. 23, 327-340.

Péron, J., Dondaine, T., Le Jeune, F., Grandjean, D., Vérin, M., 2012. Emotional processing in Parkinson's disease: a systematic review. Mov. Disord. 27, 186-199.

Pivik, R.T., Broughton, R.J., Coppola, R., Davidson, R.J., Fox, N., Nuwer, M.R., 1993. Guidelines for the recording and quantitative analysis of electroencephalographic activity in research contexts. Psychophysiology 30, 547-558.

Pfeifer, E., Novagk, R., Maess, B., 1995. Software for EEG/ERP evaluation. In: Friederici, A.D. (Ed.), Max Planck Institute of Cognitive Neuroscience Leipzig Annual Report. Max Planck Institute of Cognitive Neuroscience, Leipzig, pp. 51-55.

Puce, A., Smith, A., Allison, T., 2000. ERPs evoked by viewing facial movements. Cogn. Neuropsychol. 17, 221-239.

Recio, G., Sommer, W., Schacht, A., 2011. Electrophysiological correlates of perceiving and evaluating static and dynamic facial emotional expressions. Brain Res. 1376, 66-75.

Reitan, R.M., 1992. Trail Making Test - Manual for Administration and Scoring, 2nd ed. Reitan Neuropsychology Laboratory, South Tuscon, Arizona.

Rellecke, J., Sommer, W., Schacht, A., 2013. Emotion effects on the N170: a question of reference? Brain Topogr. 26, 62-71.

Rossion, B., Delvenne, J.F., Debatisse, D., Goffaux, V., Bruyer, R., Crommelinck, M., Guérit, J.M., 1999. Spatio-temporal localization of the face inversion effect: an event-related potentials study. Biol. Psychol. 50, 173-189.

Rossion, B., Joyce, C.A., Cottrell, G.W., Tarr, M.J., 2003. Early lateralization and orientation tuning for face, word, and object processing in the visual cortex. Neuroimage 20, 1609-1624.

Sadeh, B., Podlipsky, I., Zhdanov, A., Yovel, G., 2010. Event-related potential and functional MRI measures of face-selectivity are highly correlated: a simultaneous ERP-fMRI investigation. Hum. Brain Mapp. 31, 1490-1501.

Sato, W., Kochiyama, T., Yoshikawa, S., Naito, E., Matsumura, M., 2004. Enhanced neural activity in response to dynamic facial expressions of emotion: an fMRI study. Cogn. Brain Res. 20, 81-91.

Sprengelmeyer, R., Young, A.W., Mahn, K., Schroeder, U., Woitalla, D., Büttner, T. et al., 2003. Facial expression recognition in people with medicated and unmedicated Parkinson's disease. Neuropsychologia 41, 1047-1057.

Schendan, H.E., Amick, M.M., Cronin-Golomb, A., 2009. Role of a lateralized parietal-basal ganglia circuit in hierarchical pattern perception: evidence from Parkinson's disease. Behav. Neurosci. 123, 125-136.

St. Clair, J., Borod, J.C., Sliwinski, M., Cote, L.J., Stern, Y., 1998. Cognitive and affective functioning in Parkinson's disease patients with lateralized motor signs. J. Clin. Exp. Neuropsychol. 20, 320-327.

Starkstein, S.E., Leiguarda, R., Gershanik, O., Berthier, M., 1987. Neuropsychological disturbances in hemiparkinson's disease. Neurology 37, 1762-1764.

Suzuki, A., Hoshino, T., Shigemasu, K., Kawamura, M., 2006. Disgust-specific impairment of facial expression recognition in Parkinson's disease. Brain 129, 707-717.

Tatsch, K., Schwarz, J., Mozley, P.D., Linke, R., Pogarell, O., Oertel, W.H., et al., 1997 Relationship between clinical features of Parkinson's disease and presynaptic dopamine transporter binding assessed with [123I]IPT and single-photon emission tomography. Eur. J. Nucl. Med. 24, 415-421.

Thompson, J.C., Hardee, J.E., Panayiotou, A., Crewther, D., Puce, A., 2007. Common and distinct brain activation to viewing dynamic sequences of face and hand movements. Neuroimage 37, 966-973.

Tomer, R., Levin, B.E., Weiner, W.J., 1993. Side of onset of motor symptoms 
influences cognition in Parkinson's disease. Ann. Neurol. 34, 579-584.

Trautmann, S.A., Fehr, T., Herrmann, M., 2009. Emotions in motion: dynamic compared to static facial expressions of disgust and happiness reveal more widespread emotion-specific activations. Brain Res. 1284, 100-115.

Ventura, M.I., Baynes, K., Sigvardt, K.A., Unruh, A.M., Acklin, S.S., Kirsch, H.E., Disbrow, E.A., 2012. Hemispheric asymmetries and prosodic emotion recognition deficits in Parkinson's disease. Neuropsychologia 50, 1936-1945.

Verreyt, N., Nys, G.M., Santens, P., Vingerhoets, G., 2011. Cognitive differences between patients with left-sided and right-sided Parkinson's disease. A review. Neuropsychol. Rev. 21, 405-424.

Wechsler, D.A., 1997. Wechsler Adult Intelligence Scale-III. Psychological Corporation, New York.

Wheaton, K.J., Thompson, J.C., Syngeniotis, A., Abbott, D.F., Puce, A., 2004. Viewing the motion of human body parts activates different regions of premotor, temporal, and parietal cortex. Neuroimage 22, 277-288.

Wieser, M.J., Klupp, E., Weyers, P., Pauli, P., Weise, D., Zeller, D., et al., 2012. Reduced early visual emotion discrimination as an index of diminished emotion processing in Parkinson's disease? - Evidence from event-related brain potentials. Cortex 48, 1207-1217.

Yoshimura, N., Kawamura, M., Masaoka, Y., Homma, I., 2005. The amygdala of patients with Parkinson's disease is silent in response to fearful facial expressions. Neuroscience 131, 523-534.

Yuvaraj, R., Murugappan, M., Ibrahim, N.M., Sundaraj, K., Omar, M.I., Mohamad, K., et al., 2014. Inter-hemispheric EEG coherence analysis in Parkinson's disease: assessing brain activity during emotion processing. J. Neural Transm. 122, 237-252. 